\title{
Development and Characterization of PLGA Nanoparticles Containing 17-DMAG, an Hsp90 Inhibitor
}

\begin{abstract}
Kercia P. Cruz ${ }^{1}$, Beatriz F. C. Patricio ${ }^{2}$, Vinícius C. Pires ${ }^{1}$, Marina F. Amorim ${ }^{1}$, Alan G. S. F. Pinho ${ }^{1}$, Helenita C. Quadros ${ }^{3}$, Diana A. S. Dantas ${ }^{1}$, Marcelo H. C. Chaves ${ }^{2}$, Fabio R. Formiga ${ }^{4,5}$, Helvécio V. A. Rocha ${ }^{2}$ and Patrícia S. T. Veras ${ }^{1,6 *}$

${ }^{1}$ Laboratory of Parasite-Host Interaction and Epidemiology, Gonçalo Moniz Institute, Oswaldo Cruz Foundation (FIOCRUZ), Salvador, Brazil, ${ }^{2}$ Laboratory of Micro and Nanotechnology, Institute of Technology of Drugs (Farmanguinhos), Oswaldo Cruz Foundation (FIOCRUZ), Rio de Janeiro, Brazil, ${ }^{3}$ Laboratory of Tissue Engineering and Immunopharmacology, Gonçalo Moniz Institute, Oswaldo Cruz Foundation (FIOCRUZ), Salvador, Brazil, ${ }^{4}$ Department of Immunology, Aggeu Magalhães Institute (IAM), Oswaldo Cruz Foundation (FIOCRUZ), Recife, Brazil, ${ }^{5}$ Graduate Program in Applied Cellular and Molecular Biology, University of Pernambuco (UPE), Recife, Brazil, ${ }^{6}$ National Institute of Science and Technology of Tropical Diseases (INCT-DT), National Council for Scientific Research and Development (CNPq), Salvador, Brazil
\end{abstract}

\section{OPEN ACCESS}

Edited by:

Gildardo Rivera,

Instituto Politécnico Nacional (IPN), Mexico

Reviewed by: Andrei I. Khlebnikov, Tomsk Polytechnic University, Russia Edson Roberto Silva, University of São Paulo, Brazil

*Correspondence:

Patrícia S. T. Veras patricia.veras@fiocruz.br

Specialty section: This article was submitted to Medicinal and Pharmaceutical Chemistry, a section of the journal

Frontiers in Chemistry

Received: 21 December 2020 Accepted: 09 April 2021 Published: 13 May 2021

Citation:

Cruz KP, Patricio BFC, Pires VC, Amorim MF, Pinho AGSF,

Quadros HC, Dantas DAS,

Chaves MHC, Formiga FR, Rocha HVA and Veras PST (2021) Development and Characterization of PLGA Nanoparticles Containing

17-DMAG, an Hsp90 Inhibitor. Front. Chem. 9:644827. doi: 10.3389/fchem.2021.644827
Leishmaniasis is a spectrum of neglected tropical diseases and its cutaneous form $(\mathrm{CL})$ is characterized by papillary or ulcerated skin lesions that negatively impact patients' quality of life. Current CL treatments suffer limitations, such as severe side effects and high cost, making the search for new therapeutic alternatives an imperative. In this context, heat shock protein 90 (Hsp90) could present a novel therapeutic target, as evidence suggests that Hsp90 inhibitors, such as 17-Dimethylaminoethylamino-17-Demethoxygeldanamycin (17-DMAG), may represent promising chemotherapeutic agents against CL. As innovative input for formulation development of 17-DMAG, nano-based drug delivery systems could provide controlled release, targeting properties, and reduced drug toxicity. In this work, a double emulsion method was used to develop poly (lactic-co-glycolic acid) (PLGA) nanoparticles containing 17-DMAG. The nanoparticle was developed using two distinct protocols: Protocol 1 (P1) and Protocol 2 (P2), which differed concerning the organic solvent (acetone or dichloromethane, respectively) and procedure used to form doubleemulsions (Ultra-Turrax ${ }^{\circledR}$ homogenization or sonication, respectively). The nanoparticles produced by P2 were comparatively smaller (305.5 vs. $489.0 \mathrm{~nm})$ and more homogeneous polydispersion index ( $\mathrm{Pdl})(0.129 \mathrm{vs}$. 0.33$)$ than the ones made by $\mathrm{P} 1$. Afterward, the P2 was optimized and the best composition consisted of $2 \mathrm{mg}$ of 17-DMAG, $100 \mathrm{mg}$ of PLGA, 5\% of polyethylene glycol (PEG 8000), $1.5 \mathrm{~mL}$ of the internal aqueous phase, $1 \%$ of polyvinyl alcohol (PVA), and $4 \mathrm{~mL}$ of the organic phase. Optimized P2 nanoparticles had a particle size of $297.2 \mathrm{~nm}$ (288.6-304.1) and encapsulation efficacy of $19.35 \%$ (15.42-42.18) by the supernatant method and $31.60 \%$ (19.9-48.79) by the filter/column method. Release kinetics performed at $37^{\circ} \mathrm{C}$ indicated that $\sim 16 \%$ of the encapsulated 17-DMAG was released about to $72 \mathrm{~h}$. In a separate set of experiments, a cell uptake assay employing confocal fluorescence microscopy revealed the internalization by macrophages of P2-optimized rhodamine $\mathrm{B}$ labeled nanoparticles at $30 \mathrm{~min}, 1,2,4,6,24,48$, and $72 \mathrm{~h}$. Collectively, our results indicate 
the superior performance of P2 concerning the parameters used to assess nanoparticle development. Therefore, these findings warrant further research to evaluate optimized 17-DMAG-loaded nanoparticles (NP2-17-DMAG) for toxicity and antileishmanial effects in vitro and in vivo.

Keywords: leishmaniasis, Hsp90, 17-DMAG, double emulsion, PLGA, nanoparticles

\section{INTRODUCTION}

Constituting a severe public health problem throughout the world, the spectrum of leishmaniasis consists of neglected tropical diseases caused by parasite species of the genus Leishmania, 20 of which are capable of infecting humans (Masmoudi et al., 2013; Akhoundi et al., 2016; WHO, 2020a). Although endemic in 97 countries, the disease is mainly concentrated in Africa, Asia, and the Americas (WHO, 2020b). It is currently estimated that around 12 million people are infected worldwide, with an annual incidence of more than one million new cases per year; one billion of the world's population lives in areas at risk of infection (Akhoundi et al., 2016; WHO, 2020a).

Leishmaniasis can be divided into two primary clinical forms: cutaneous and visceral, with varying presentations depending on the species and virulence of the infecting parasite, as well as the type of host immune response (Kaye and Scott, 2011; Akhoundi et al., 2016; Oryan and Akbari, 2016; Srivastava et al., 2016; Veras and De Menezes, 2016; WHO, 2020a). Among the cutaneous presentations, mucocutaneous leishmaniasis (MCL), caused mainly by $L$. aethiopica in the Old World and $L$. braziliensis in the New World, is the most debilitating form, with destructive lesions occurring on the palate, lips and nasal septum (Akhoundi et al., 2016; Burza et al., 2018; WHO, 2020a). The most common form, localized cutaneous leishmaniasis (LCL), is caused by a variety of parasite species, including $L$. major, $L$. tropica, and L. aethiopica in the Old World, in addition to $L$. braziliensis, L. guyanensis, L. amazonensis, and L. mexicana in the New World (Kaye and Scott, 2011; Masmoudi et al., 2013; Burza et al., 2018; Meira and Gedamu, 2019). Despite not being fatal, LCL can affect patients' quality of life according to the evolution and spread of skin lesions, social stigmatization, psychological effects, and absenteeism (Carvalho et al., 1994; Scorza et al., 2017; Burza et al., 2018).

Currently, chemotherapy is the recommended treatment for patients diagnosed with leishmaniasis, mainly pentavalent antimonials and Amphotericin B in a free or liposomalencapsulated form (Croft et al., 2006; Frézard et al., 2009; Seifert, 2011; Brasil, 2015; De Menezes et al., 2015; AndradeNeto et al., 2018). Alternatively, other drugs, such as pentamidine and paromomycin, can also be applied in leishmaniasis treatment (Santos et al., 2008; De Menezes et al., 2015). These therapies present several limitations, including high cost, invasive route of administration, prolonged cycle and systemic side effects, e.g., weakness, myalgia, rigors/chills, hemolysis and fever, as well as instability at high temperatures in some formulations (Sundar et al., 2004; Frézard et al., 2009; Chávez-Fumagalli et al., 2015; De Menezes et al., 2015). Additionally, drug accumulation in the organs can lead to pancreatitis, nephrotoxicity, hepatotoxicity, myocarditis, and cardiotoxicity (Rath et al., 2003; Frézard et al., 2009; Seifert, 2011; Masmoudi et al., 2013; Chávez-Fumagalli et al., 2015; De Menezes et al., 2015). The only currently available non-invasive orally administered treatment for leishmaniasis, miltefosine, presents limitations including vomiting, diarrhea, kidney, and liver toxicity, as well as potential teratogenic effects and high cost in some regions (Rath et al., 2003; Seifert, 2011; Masmoudi et al., 2013; De Menezes et al., 2015; Andrade-Neto et al., 2018). This scenario highlights the need to discover new drugs that offer increased efficacy and less toxicity (De Menezes et al., 2015). So far, efforts to this end have focused on (i) increasing the safety and efficacy of treatments already in use; (ii) combined drug therapy via novel therapeutic protocols; (iii) the search for new therapeutic targets in parasites or host cells; (iv) repurposing drugs used to treat other diseases; (v) developing more effective delivery systems (Frézard et al., 2009; De Menezes et al., 2015; Andrade-Neto et al., 2018).

Heat Shock Protein 90 (Hsp90), a ubiquitous and highly conserved molecular chaperone, is responsible for performing the folding of other proteins, namely client proteins, subsequently preventing the post-translational formation of oligomeric complexes with incorrect, inactive and nonfunctional structures (Zhao and Houry, 2005; Erlejman et al., 2014). This chaperone has been described as a potential therapeutic target in treating cancer and infectious diseases caused by different parasite species, including those of the Leishmania genus (Solit and Chiosis, 2008; Pallavi et al., 2010; Roy et al., 2012; Whitesell and Lin, 2012; Schopf et al., 2017; Guswanto et al., 2018). Notably, in leishmaniasis, Hsp90 has been shown to aid in re-establishing the functional stability of proteins in response to environmental pressure, such as differences in $\mathrm{pH}$ and temperature, during parasite differentiation processes (Zilberstein and Shapira, 1994; Graefe et al., 2002; Solit and Chiosis, 2008; Pallavi et al., 2010; Roy et al., 2012; Hombach et al., 2014; Schopf et al., 2017).

Structurally, Hsp90 is comprised of three main domains: the intermediate central proteolytic domain, involved in the interface between Hsp90 and its client proteins; the C-terminal domain, which facilitates homodimerization; the N-terminal domain, responsible for interaction with and the hydrolysis of ATP (Pratt and Toft, 2003; Zhao and Houry, 2005; Brown et al., 2007). The family of benzoquinone ansamycins constitutes a class of Hsp90 inhibitors that compete with ATP for binding at the Hsp90 interaction site, thereby hindering chaperone activity (Zhao and Houry, 2005; Brown et al., 2007; Erlejman et al., 2014). Subsequently, truncated or malformed proteins become degraded by the ubiquitin-proteasome system (Chiosis et al., 
2004; Xiao et al., 2006; Sidera and Patsavoudi, 2013). In parasites of the genus Leishmania, this inhibition leads to parasite death, evidencing the importance of Hsp90 in the maintenance of cellular homeostasis (Wiesgigl et al., 2001; Li et al., 2009; Roy et al., 2012; Hombach et al., 2013).

Research by our group previously demonstrated that geldanamycin (GA), 17-AAG, and 17-DMAG, Hsp90 inhibitors of benzoquinone ansamycin family, were capable of eliminating promastigote forms of $L$. amazonensis at concentrations determined as non-toxic for human monocyte lineage cells (THP-1) (Palma et al., 2019). It was also demonstrated that the treatment of $L$. amazonensis-infected macrophages with 17-AAG reduced the percentage of infected macrophages and numbers of intracellular parasites in a time- and dose-dependent manner at concentrations deemed non-toxic to host cells (Petersen et al., 2012). Furthermore, 17-AAG was found to control, both in vitro and in vivo, L. braziliensis infection in BALB/c mouse macrophages (Santos et al., 2014). This study demonstrates that 17-AAG reduces the size of ear lesions and parasitic load at the lesion site, but not in the draining lymph nodes of infected mice, resulting in infection relapse (Santos et al., 2014). To overcome this described limitation, it will be tested a water-soluble analog of 17-AAG, 17-DMAG (Egorin et al., 2002; Sausville, 2004; Whitesell and Lin, 2012). Because it is a water-soluble molecule and has better pharmacokinetics than 17-AAG, 17-DMAG can achieve lymph nodes of treated animals, eliminating the parasites on this site. To optimize a formulation containing-Hsp90 inhibitor for leishmaniasis treatment, we propose the encapsulation of 17-DMAG in a nanoparticle, which can have controlled release of the drug, prolonging its action with fewer administrations. This delivery system can also help prevent toxicity occurrences in future tests for L. braziliensis infection control.

Nanoparticle-based controlled release systems offer several advantages: improved safety, efficacy, target specificity (drug targeting), biocompatibility, bioavailability, biodegradability, and reduced toxicity in comparison to traditional drug delivery systems (Zhang et al., 2008; Formiga et al., 2009; Yildirimer et al., 2011; Lin, 2015; Utreja et al., 2020). By directing the active principle to specific tissues and releasing it gradually over time, the dose necessary to observe treatment efficacy becomes reduced, thereby contributing to a reduction in side effects (Yildirimer et al., 2011; Wolfram et al., 2015). Synthetic polymers such as poly(lactic-co-glycolic acid) (PLGA), polylactic acid (PLA) and polycaprolactone (PCL) are commonly used in drug delivery systems because they do not offer a risk of inducing an unwanted immune response (Formiga et al., 2009; Zhang and Zhang, 2017; Utreja et al., 2020). The main application of these systems consists of cases in which the free form of a drug presents limitations, such as shortened half-life, requiring the need for multiple applications, and inadequate target specificity, which can lead to the occurrence of a range of side effects (Zhang et al., 2008; Formiga et al., 2009). Thus, the present work aimed to produce polymeric nanoparticles (NPs) containing the Hsp90 inhibitor, 17-DMAG, and perform morphological and physical-chemical characterization. The results obtained herein will enable, in the future, an evaluation of in vitro and

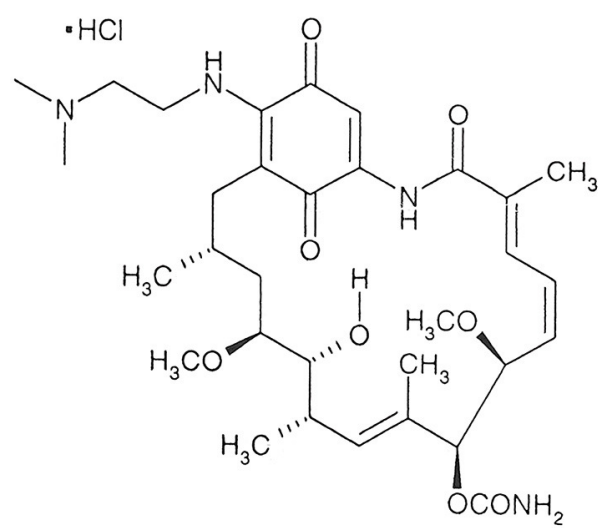

FIGURE 1 | Chemical structure of 17-DMAG. Extracted from 17-DMAG datasheet (LC Laboratories)

in vivo optimized 17-DMAG-loaded NPs (NP2-17-DMAG) as antileishmanial treatment.

\section{MATERIALS AND METHODS}

\section{Reagents and Chemicals}

The hydrochloride salt of alvespimycin, 17-DMAG (Figure 1), was purchased from LC Laboratories (Massachusetts, USA). Resomer $®$ RG 503 H, Poly (D, L-lactide-co-glycolide) (50:50) (PLGA), Poly (vinyl alcohol) (87-90\% hydrolyzed average mol wt 30,000-70,000, PVA) and poly(ethylene glycol) (PEG, MW $=8,000$ ) were purchased from Sigma-Aldrich (Darmstadt, Germany). Alamar blue ${ }^{\circledR}$ was purchased from Invitrogen (Massachusetts, USA). Microcon-30 0.5ML microtubes were purchased from Merk Millipore (Darmstadt, Germany). A C18 HPLC column and C18 Supelguard Guard Cartridge were purchased from Sigma-Aldrich (Darmstadt, Germany).

\section{Preparations of PLGA NPs}

Two different double emulsion protocols were used to prepare PLGA NPs. In the first protocol (P1), PLGA NPs containing 17DMAG (NP1-17-DMAG) were prepared using a modified double emulsion/solvent evaporation technique (Salvador et al., 2015). Briefly, $50 \mathrm{mg}$ of PLGA was dissolved in $5 \mathrm{~mL}$ of acetone, and $2 \mathrm{mg}$ of 17-DMAG was dissolved in 5\% PEG solution $(250 \mathrm{mg}$ in $5 \mathrm{~mL}$ of distilled water). The 17-DMAG solution was then added to the pre-cooled PLGA solution and emulsified using a Q700 sonicator (QSonica, Newtown, Connecticut, USA) at $6 \%$ amplitude for $2 \mathrm{~min}$. Subsequently, $10 \mathrm{~mL}$ of PVA $1 \% \mathrm{w} / \mathrm{v}$ was added to the mixture. The recipient was then covered with aluminum foil and then homogenized at 10,000 rpm for $5 \mathrm{~min}$ (Ultra-turrax ${ }^{\circledR}$ T-25, IKA, Germany) to form a double emulsion. Next, $10 \mathrm{~mL}$ of $2 \%$ isopropyl alcohol was added. Finally, the suspension was magnetically stirred for $30 \mathrm{~min}$ with a subsequent solvent evaporation step using a rotary evaporator (IKA) for $1 \mathrm{~h}$ at $56^{\circ} \mathrm{C}$ under $250 \mathrm{mBar}$.

In the second double emulsion protocol (P2), PLGA NPs (NP2-17-DMAG) were also produced using another double 
emulsion/solvent evaporation technique (Mainardes et al., 2009). Initially, $100 \mathrm{mg}$ of PLGA was dissolved in $4 \mathrm{~mL}$ of dichloromethane. Another solution containing $2 \mathrm{mg}$ of $17-$ DMAG dissolved in $1.5 \mathrm{~mL}$ of PEG $5 \% \mathrm{w} / \mathrm{v}$ was then added to the pre-cooled PLGA solution to form a single emulsion using a Q700 sonicator (QSonica, Newtown, Connecticut, USA) at $40 \%$ amplitude for $1 \mathrm{~min}$. To form the double emulsion, the single emulsion was incorporated in $10 \mathrm{~mL}$ of PVA $1 \% \mathrm{w} / \mathrm{v}$, then sonicated. Finally, $30 \mathrm{~mL}$ of PVA $1 \% \mathrm{w} / \mathrm{v}$ was added to the recipient, covered with aluminum foil and magnetically stirred for $30 \mathrm{~min}$. The solvent extraction step was performed using a rotary evaporator (IKA) for $1 \mathrm{~h}$ at $40^{\circ} \mathrm{C}$ under $200 \mathrm{mBar}$.

Following each nanoparticle protocol preparation, suspensions were washed in distilled water three times at $39,800 \times \mathrm{g}$ for $15 \mathrm{~min}$ at $4^{\circ} \mathrm{C}(\mathrm{T}-865$, Thermo Fisher Scientific, Massachusetts, USA). Samples were frozen at $-80^{\circ} \mathrm{C}$, then lyophilized for $24 \mathrm{~h}$ at $-48^{\circ} \mathrm{C}$ under $0.050 \mathrm{mBar}$ (FreeZone 2.5 Liter Benchtop, Labconco, USA) and subsequently stored at $4^{\circ} \mathrm{C}$. For blank nanoparticle preparation (NP1- $\varnothing$ and NP2- $\varnothing$ ), these same protocols were followed in the absence of 17-DMAG.

To evaluate the influence of PLGA and PEG concentration on the encapsulation efficiency (\%EE) of 17-DMAG and the size of the produced NPs, P2 was performed as described above using 100 or $200 \mathrm{mg}$ of PLGA and $2.5 \% \mathrm{w} / \mathrm{v}$ or $5 \% \mathrm{w} / \mathrm{v}$ of PEG.

\section{PLGA NPs Characterization Dynamic Light Scattering}

DLS was used to measure the particle size, polydispersion index (PdI) and zeta potential (ZP) of the obtained NPs. After washing, NP-17-DMAG or NP- $\varnothing$ produced by the P1 or P2 doubleemulsion protocols were resuspended in $5 \mathrm{~mL}$ of distilled water and diluted 1:125 in distilled water. DLS analysis was performed in triplicate using a ZetaSizer Nano ZS90 (Malvern Panalytical, $\mathrm{UK})$ at $25^{\circ} \mathrm{C}$.

\section{Transmission Electron Microscopy}

For imaging and size confirmation, NPs were washed as described in item 2.2 and analyzed by TEM. Aliquots of NP-17-DMAG or NP- $\varnothing$ produced by the $\mathrm{P} 1$ or $\mathrm{P} 2$ double-emulsion protocols were diluted 1:10 in distilled water, then $10 \mu \mathrm{L}$ of each sample was placed on a formvar film-coated grid and stained with $2 \%$ uranyl acetate for $2 \mathrm{~min}$. TEM analysis was performed using a JEM-1230 transmission electron microscope (JEOL LTD, Japan).

\section{Scanning Electron Microscopy}

SEM was used to examine the shape and surface morphology of the NP-17-DMAG or NP- $\varnothing$ produced by the P1 or P2 doubleemulsion protocols. For SEM analysis, lyophilized NPs (1 mg) were placed on an adhesive stub and coated with gold-palladium under vacuum using an ion coater. All samples were analyzed and photographed at $15 \mathrm{kV}$ using a JSM-6390LV microscope (JEOL LTD, Japan).

\section{HPLC for 17-DMAG Quantification}

To quantify 17-DMAG, HPLC was performed using a C18 HPLC column and C18 Supelguard Guard Cartridge following manufacturer protocols. First, the mobile phase was prepared using HPLC grade acetonitrile (27\%), HPLC grade methanol (27\%), ultrapure water (46\%), and trifluoroacetic acid (0.05\%). In parallel, 17-DMAG was diluted in distilled water at an initial concentration of $500 \mu \mathrm{g} / \mathrm{mL}$, then diluted from 50 to $1 \mu \mathrm{g} / \mathrm{mL}$ in the mobile phase to construct concentration curves in triplicate. Each sample was analyzed at a 2D wavelength of $254 \mathrm{~nm}$ for $8 \mathrm{~min}$ at $25^{\circ} \mathrm{C}$. The mobile phase flow rate was $1 \mathrm{~mL} / \mathrm{min}$, with $10 \mu \mathrm{L}$ of each sample injected. The observed retention time for 17-DMAG under these conditions was $\sim 3.8 \mathrm{~min}$. Nanoparticle concentrations of 17-DMAG were calculated using a free compound curve (Empower software, version 3).

\section{Encapsulation Efficiency (\%EE) of 17-DMAG in NPs}

Encapsulation efficiency determination was performed using two indirect methods: filter/column or supernatant. After the solvent was evaporated using the filter/column method, $500 \mu \mathrm{L}$ of nanoparticle suspension was centrifuged in a $1.5 \mathrm{~mL}$ microtube with a Microcon 30 filter under $14,000 \times \mathrm{g}$ for $1 \mathrm{~h}$ at $4^{\circ} \mathrm{C}$. As the free drug fraction (F) in each sample flowed through the filter, NPs were retained. In parallel, absolute ethanol was added to another $500 \mu \mathrm{L}$ aliquot of total nanoparticle suspension at a proportion of $1: 1$ to determine the total amount of 17 DMAG. After centrifugation at $6,200 \times \mathrm{g}$ for $15 \mathrm{~min}$ at $4^{\circ} \mathrm{C}$, the supernatant was collected and the total quantity of $17-\mathrm{DMAG}$ (T) was measured. \%EE was evaluated by HPLC following the formula:

$$
\% E E=\frac{(T-F)}{T} \times 100,
$$

where T corresponds to the total mass of the drug in the sample, whether encapsulated or not, and F corresponds to the nonencapsulated fraction (free fraction).

For the supernatant method, the supernatants obtained from three washes of the produced NPs were collected and then diluted at 1:5 in the mobile phase. HPLC then evaluated the $\% \mathrm{EE}$ according to the formula:

$$
\% E E=\frac{S}{T} \times 100
$$

where $S$ corresponds to the total mass of $17-\mathrm{DMAG}$ in the supernatants and $\mathrm{T}$ is the total mass of the drug added for encapsulation.

\section{Release of 17-DMAG From NP2-17-DMAG in vitro}

The release of 17-DMAG from NP2-17-DMAG was assessed in vitro using a modified method to determine the release kinetic profile (Quadros et al., 2020). Briefly, $2 \mathrm{mg}$ of NP2-17DMAG were placed into $1.5 \mathrm{~mL}$ polypropylene microcentrifuge tubes and resuspended in $1 \mathrm{~mL}$ of Dulbecco's modified Eagle's medium (DMEM) (Gibco), supplemented with $20 \mathrm{mM}$ of HEPES (Sigma), $42.14 \mathrm{mM}$ of sodium bicarbonate (Sigma), $10 \%$ of inactivated fetal bovine serum (Gibco), $2 \mathrm{mM}$ of glutamine (Sigma), and $20 \mu \mathrm{g} / \mathrm{mL}$ of ciprofloxacin (Isofarma, Precabura, CE, BR) (complete DMEM medium). Next, the sealed tubes were 
placed in a rotating shaker and maintained at $37^{\circ} \mathrm{C}$ for $72 \mathrm{~h}$. At each specific time point $(1,3,6,12,24,48$, and $72 \mathrm{~h})$, the sample tubes were removed from the incubator and centrifuged at 21,000 $\times \mathrm{g}$ for $15 \mathrm{~min}$ at $4^{\circ} \mathrm{C}$. The supernatant was then collected, frozen and immediately replaced with an equal volume of fresh release medium. To determine the 17-DMAG concentration, collected supernatants were diluted in the mobile phase and quantified using HPLC Shimadzu LC20-A (São Paulo, Brazil). All assays were performed in triplicate.

\section{Animal Manipulation and Ethics Statement}

$\mathrm{BALB} / \mathrm{c}$ mice, male or female, aged 6-12 weeks, were provided by the Gonçalo Moniz Institute (IGM/FIOCRUZ) Animal Care Facility. The animals were maintained under pathogen-free conditions, with food and water ad libitum. All procedures involving animals were conducted under the International Guiding Principles for Biomedical Research Involving Animals. The Institutional Review Board approved this study's experimental design (CEUA protocol no. 007/2020) of the Gonçalo Moniz Institute, Bahia-Brazil (IGM-FIOCRUZ/BA).

\section{Obtainment of Bone Marrow-Derived Macrophages From BALB/c Mice}

$\mathrm{BALB} / \mathrm{c}$ mice were euthanized using thiopental intraperitoneal injection $(50 \mathrm{mg} / \mathrm{kg})$. Mouse femurs and tibias were aseptically removed and kept in cold $0.9 \% \mathrm{NaCl}$ solution containing $0.01 \mathrm{mg} / \mathrm{mL}$ of ciprofloxacin. In a sterile environment, bone extremities were removed and marrow cells were extracted by washing the bone cavity with Roswell Park Memorial Institute (RPMI) 1640 medium (GIBCO) supplemented with $20 \mathrm{mM}$ of HEPES (SIGMA), $23 \mathrm{mM}$ of sodium bicarbonate (SIGMA), 10\% of inactivated fetal bovine serum (Gibco), $2 \mathrm{mM}$ of Glutamine (Sigma) and $20 \mu \mathrm{g} / \mathrm{mL}$ of ciprofloxacin (Isofarma, Precabura, CE, BR) (complete RPMI medium). Extracted marrow cells were centrifuged at $300 \times \mathrm{g}$ at $4^{\circ} \mathrm{C}$ for $10 \mathrm{~min}$, then resuspended and cultivated in Petri dishes (three plates per animal) containing $10 \mathrm{~mL}$ of complete RPMI medium with $30 \%$ supernatant from L929 cell culture containing granulocyte macrophage colonystimulating factor (GM-CSF). The dishes were cultivated at $37^{\circ} \mathrm{C}$ under $5 \% \mathrm{CO}_{2}$ and $95 \%$ humidity for $24 \mathrm{~h}$, after which the supernatant was transferred to new plates. After $72 \mathrm{~h}$, an additional $5 \mathrm{~mL}$ of complete RPMI medium containing 30\% L929 supernatant was added to each culture to re-stimulate cells for differentiation.

On the 7th day, BMDM were recovered from bacterial Petri dishes using $5 \mathrm{~mL}$ of $1 \mathrm{mM}$ EDTA solution ( $\mathrm{pH} \mathrm{8.0)}$ for $5 \mathrm{~min}$ at $37^{\circ} \mathrm{C}$. Cells were centrifuged at $300 \times \mathrm{g}$ for $10 \mathrm{~min}$ at $4^{\circ} \mathrm{C}$, then resuspended in $1 \mathrm{~mL}$ of complete DMEM medium and counted in a Neubauer chamber.

\section{Uptake of Fluorescent NPs by BMDM in vitro}

NPs containing rhodamine B (Sigma) were produced using the P2 protocol (item 2.2). BMDM were obtained as described above and plated at $10^{5}$ cells per well in $1 \mathrm{~mL}$ of complete DMEM medium on 24-well plates containing glass coverslips. For the in vitro uptake assay, rhodamine B-containing NPs were lyophilized and then incubated with BMDM for $30 \mathrm{~min}$,
TABLE 1 | Particle size (Size), polydispersion index (Pdl), zeta potential (ZP) and encapsulation efficiency (\%EE) by supernatant of filter/column methods of NPs produced by P1 or P2 double emulsion protocols.

\begin{tabular}{lccccc}
\hline Protocol & Size (nm) & Pdl & ZP (mV) & $\begin{array}{c}\text { \%EE } \\
\text { (supernatant) }\end{array}$ & $\begin{array}{c}\text { \%EE (filter } \\
\text { /column) }\end{array}$ \\
\hline P1 & 489 & 0.33 & -34.4 & $14.9 \%$ & $14.02 \%$ \\
P2 & 305.5 & 0.129 & -28.7 & $15.04 \%$ & $17 \%$ \\
\hline
\end{tabular}

$1,2,4,6,24,48$, and $72 \mathrm{~h}$. Wells were washed at each time point, and cells were fixed with $4 \%$ paraformaldehyde (PFA) for $15 \mathrm{~min}$ at room temperature. Finally, coverslips were mounted on slides using ProLong Gold antifade with DAPI ${ }^{\circledR}$ (Invitrogen, Darmstadt, Germany). Images were obtained by confocal fluorescence microscopy using a Leica SP8 device (Leica Microsystems, Mannheim, Germany).

\section{Statistical Analysis}

Graphs were constructed and statistical analyses were performed using GraphPad Prism version 5.01 (GraphPad Software Inc). The Kolmogorov-Smirnov test was employed to verify normality. For data with Gaussian distribution, Student's $t$-test or one-way ANOVA were used to compare between two groups or among three or more groups, respectively, followed by Tukey's posttest. For non-gaussian distributions, the Mann-Whitney U test was applied for comparisons between two groups, while KruskalWallis was used to compare three or more groups. Differences were considered statistically significant when $p<0.05$.

\section{RESULTS}

\section{DLS Characterization and \%EE of NPs Produced by P1 or P2 Double Emulsion Protocols}

The NP1-17-DMAG had a larger average size, size variation (PdI), and ZP than the NP2-17-DMAG (Table 1). No differences were detected in \%EE values regardless of the quantitation method (supernatant or filter/column) used to determine the amount of 17-DMAG encapsulated in NP1-17-DMAG or NP217-DMAG (Table 1).

\section{Morphological Characterization of NP1 and NP2}

Consistent with the obtained PdI values, electron microscopy analysis revealed a more significant size variation in NP1 (Figures 2A,B) compared to NP2 (Figures 2C,D). Both protocols produced spherical, regular-shaped NPs (Figure 2). No morphological differences were observed between NP- $\varnothing$ and NP-17-DMAG.

SEM morphological analysis confirmed the spherical shape and smooth surface of the NPs produced by both double emulsion protocols (Figure 3). Consistent with DLS and TEM results, SEM analysis also revealed that NP1- $\varnothing$ and NP1-17-DMAG exhibited more considerable size variation (Figures 3A,B) compared to NP2- $\varnothing$ and NP2-17-DMAG (Figures 3C,D). Again, no morphological differences were 


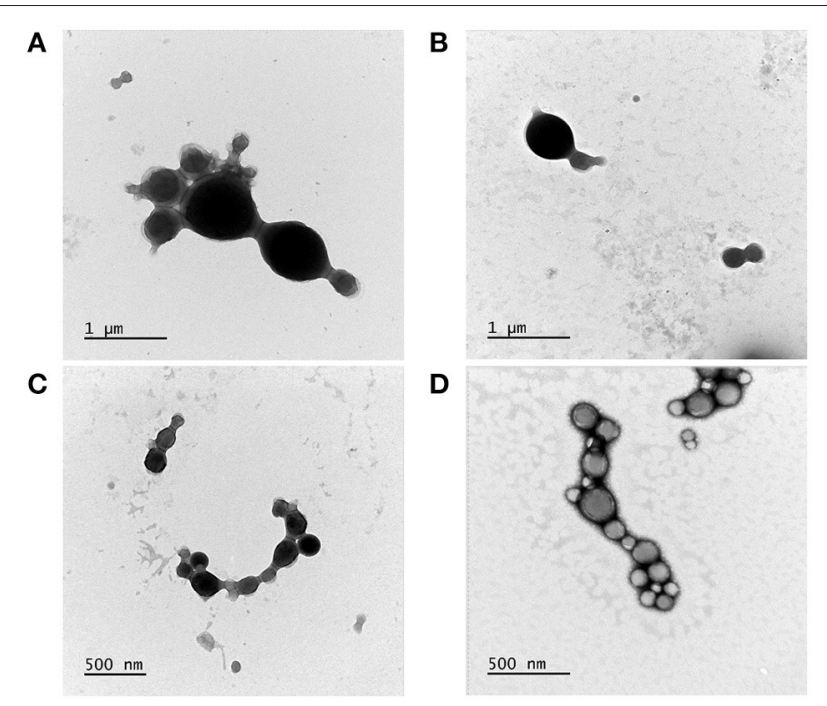

FIGURE 2 | Morphological characterization of NP1 and NP2 by TEM Transmission electron microscopy images of NP- $\varnothing(\mathbf{A}, \mathbf{C})$ and NP-17-DMAG (B,D) produced by P1 (A,B) or P2 (C,D) double emulsion protocols. $10 \mu \mathrm{L}$ of each sample was contrasted with $2 \%$ uranyl acetate for 2 min in a formvar grid. Bars represent size references in $\mu \mathrm{m}$ or $\mathrm{nm}$.
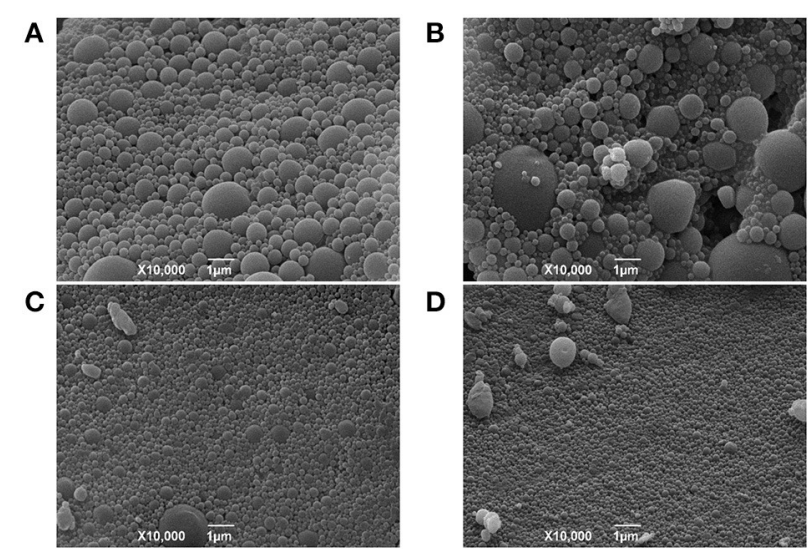

FIGURE 3 | SEM evaluations of NP1 and NP2. NP-Ø (A,C) or NP-17-DMAG (B,D) were produced by P1 (A,B) or P2 (C,D) double emulsion protocols. The produced NPs were frozen at $-80^{\circ} \mathrm{C}$ and lyophilized for $24 \mathrm{~h}$ at $-48^{\circ} \mathrm{C}$ under 0.050 mbar. Approximately $1 \mathrm{mg}$ of each sample was vacuum-coated with gold-palladium using an ion coater and analyzed by SEM. All samples were analyzed and photographed at $15 \mathrm{kV}$. Bars represent $1 \mu \mathrm{m}$.

observed between NP- $\varnothing$ (Figures 3A,C) and NP-17-DMAG (Figures 3B,D) regardless of the protocol used.

\section{P2 Optimization Protocol}

As NP2-17-DMAG exhibited superior physical-chemical and morphological characteristics compared to NP1-17-DMAG, we employed P2 to optimize the 17-DMAG encapsulation process with some variations. NPs produced using 5\% PEG presented a smaller median size of 297.2 nm (Q1: 288.6; Q2:304.1) compared to those made using 2.5\% PEG (median size: $336.5 \mathrm{~nm}$; Q1: 318.4;
A

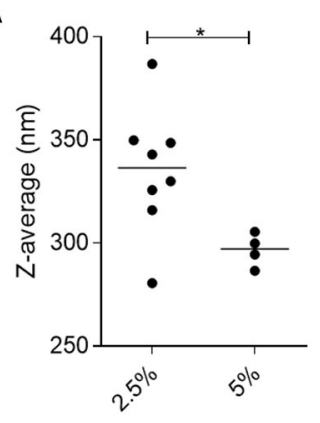

C

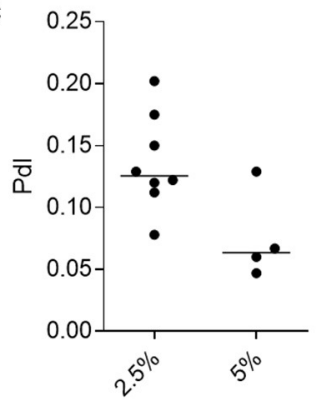

E

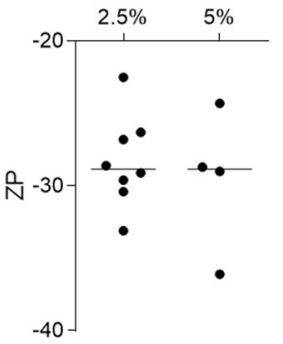

B

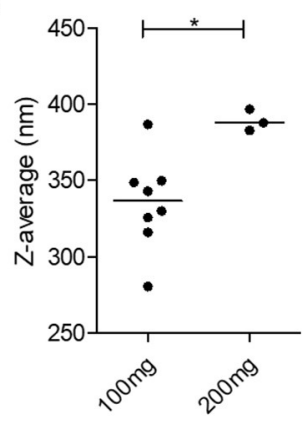

D



$\mathbf{F}$

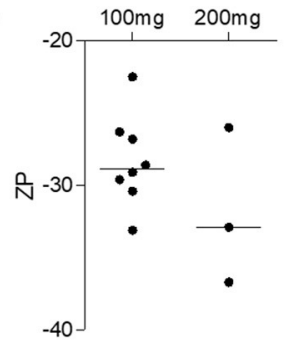

FIGURE 4 | Evaluation of the influence of amount of PLGA and PEG concentration on nanoparticle physiochemical features in NP2-17-DMAG. DLS evaluated the effect of varying amounts of PEG $(\mathbf{A}, \mathbf{C}, \mathbf{E})$ and PLGA $(\mathbf{B}, \mathbf{D}, \mathbf{F})$ on the size (A,B), Pdl (C,D), and ZP (E,F) of NP2-17-DMAG. Each point represents an individual experiment. Mann-Whitney test, ${ }^{*} p<0.05$.

Q2: 349.6) (Figure 4A). Similarly, NPs produced containing $100 \mathrm{mg}$ of PLGA presented a smaller median size of $336.5 \mathrm{~nm}$ (Q1: 318.4; Q2: 349.6) in comparison to those containing $200 \mathrm{mg}$ of PLGA (median size: $387.8 \mathrm{~nm}$; Q1: 382.8; Q2: 396.7) (Figure 4B). No differences were detected concerning PdI and ZP values among NPs prepared using different PEG concentrations (Figures 4C,E), nor different PLGA masses (Figures 4D,F).

Similar \%EE values were found for NP2-17-DMAG regardless of the amount of PEG used (2.5 or 5\%) (Figure 5A). Median \%EE values for 2.5 and 5\% of PEG were 23.51\% (Q1: 19.66; Q2: 27.38) and 19.35\% (Q1: 15.42; Q2: 42.18), respectively, using the supernatant analysis method, vs. $34.12 \%$ (Q1: 29.31; Q2: 36.73) and 31.60\% (Q1: 19.90; Q2: 48.79) using the filter/column method (Figure 5A). Furthermore, similar \%EE results were seen in NPs produced with different amounts of PLGA (Figure 5B) using the supernatant quantitative analysis 

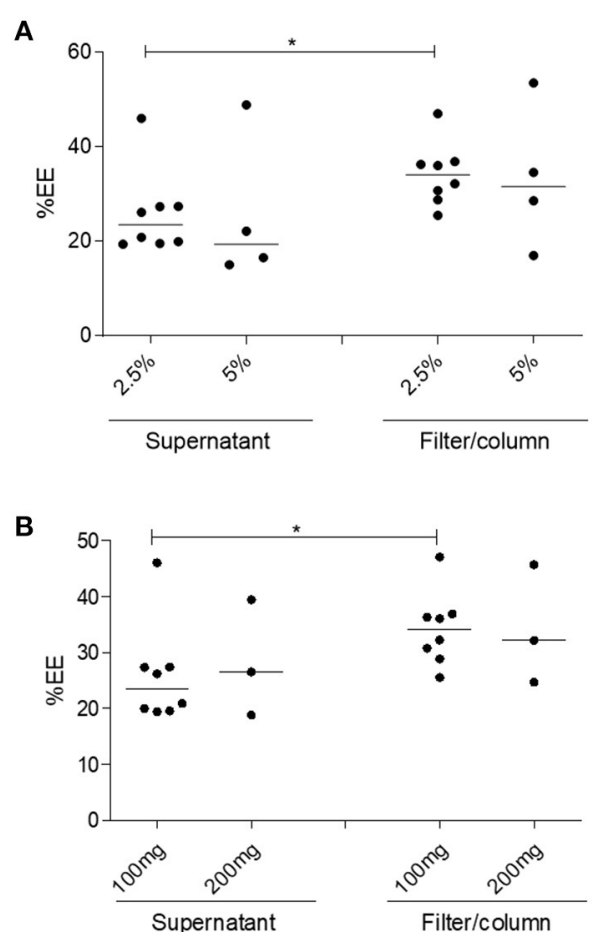

FIGURE 5 | Evaluation of the influence of PLGA and PEG concentration on the \%EE of 17-DMAG in NP2-17-DMAG. The effect of varying amounts of PEG (A) and PLGA (B) on the \%EE of 17-DMAG in NP2-17-DMAG was determined by filter/column or supernatant analysis methods as described in section Material and Methods. Measurements were performed using HPLC. Each point represents an individual experiment. Mann-Whitney test, ${ }^{*} p<0.05$.

method, with respective median values of 23.51\%(Q1: 19.66; Q2: 27.38) and 26.48\% (Q1: 18.78; Q2: 39.39) for 100 and $200 \mathrm{mg}$ of PLGA, respectively. The filter/column method yielded median \%EE values of 34.12\% (Q1: 29.31; Q2: 36.73) for $100 \mathrm{mg}$ and 32.12\% (Q1: 24.66; Q2: 44.63) for $200 \mathrm{mg}$ of this polymer. It is worth noting that, in comparison to the supernatant analysis method, higher \%EE results were obtained using the quantitative filter/column method regardless of NP2 protocol modifications (Figure 5).

\section{In vitro Release of 17-DMAG From NP2-17-DMAG}

At $1,3,6,12,24,48$, and $72 \mathrm{~h}$ of incubation, 5.36, 7.86, 9.85, 11.64, $13.41,14.36$, and $16 \%$ of $17-D M A G$ were cumulatively released from NP2-17-DMAG (Figure 6) in vitro, i.e., the amount of 17-DMAG was observed to continuously increase in complete DMEM medium for $72 \mathrm{~h}$ (Figure 6).

\section{Uptake of Fluorescent NPs by BMDM in vitro}

The rhodamine-encapsulated NPs produced by P2 (NP2rhodamine) presented similar size, shape, and appearance (smooth surface) as the morphological characteristics of NPs produced with or without 17-DMAG (data not shown). The

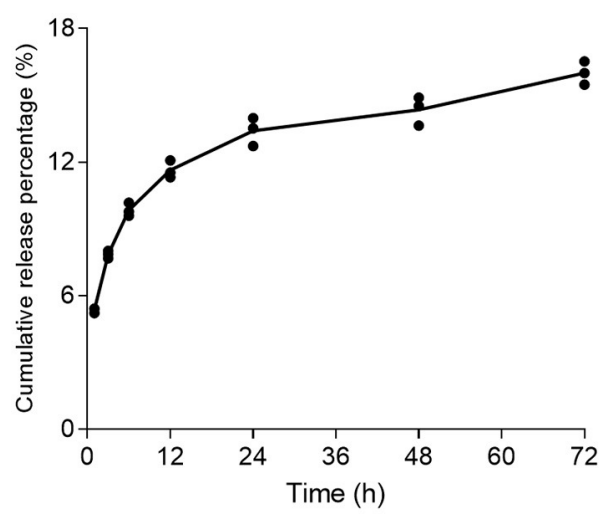

FIGURE 6 | Kinetics of 17-DMAG release from NP2-17-DMAG. NP2-17-DMAG were lyophilized and incubated in complete DMEM medium under rotary agitation at $37^{\circ} \mathrm{C}$. After $1,3,6,12,24,48$ or $72 \mathrm{~h}$, samples were centrifuged, the supernatant was collected, and the medium was replaced. The release of 17-DMAG was quantified in supernatants using HPLC. Mean, $n=3$.

uptake of NP2-rhodamine by BMDM was observed at an early incubation time of $30 \mathrm{~min}, 1,2,4$, and $6 \mathrm{~h}$ (Figures $7 \mathrm{~A}-\mathrm{E}$, respectively). After 24,48 or $72 \mathrm{~h}$ (Figures $7 \mathbf{F}-\mathbf{H}$, respectively), greater numbers of NPs were observed in the cytoplasm of BMDM, indicating continued uptake by these cells.

\section{DISCUSSION}

The present work encapsulated 17-DMAG in PLGA NPs and investigated the resulting physical-chemical, morphological, and biological parameters. To standardize NP production, two double emulsion-solvent evaporation protocols (P1 and P2) were used (Astete and Sabliov, 2006; Mainardes et al., 2009). Analyses by DLS, TEM and SEM showed that NP1 presented larger sizes and higher PdI than NP2, which agrees with a previous report (Astete and Sabliov, 2006). We found that modifications in the PLGA-NP production protocol, such as the nature of the organic solvent used or the emulsion method, altered the characteristics of the produced NP. The larger sizes presented by NP1 may be due to laminar flow under stirring, such as that produced by the Ultra-Turrax $®$ dispersing device. Accordingly, monodispersed drops may form in the emulsion, increasing the size of the produced NP, which is not observed under sonication (Astete and Sabliov, 2006). Concerning ZP, a measurement of the electrical behavior of NPs, both protocols produced NPs with values around $-30 \mathrm{mV}$, indicating stability with minimal aggregate formation (Formiga et al., 2009). Furthermore, the observed similarity between \%EE values in each protocol can be justified by using the same polymer and surfactant (PEG), as these parameters significantly influence the entrapment of hydrophilic drugs (Astete and Sabliov, 2006).

The morphological characterization of NP1 and NP2 by TEM and SEM confirmed differences in size and PdI between the two protocols, revealing a similarly regular, spherical shape and smooth surface appearance. These aspects are essential as 


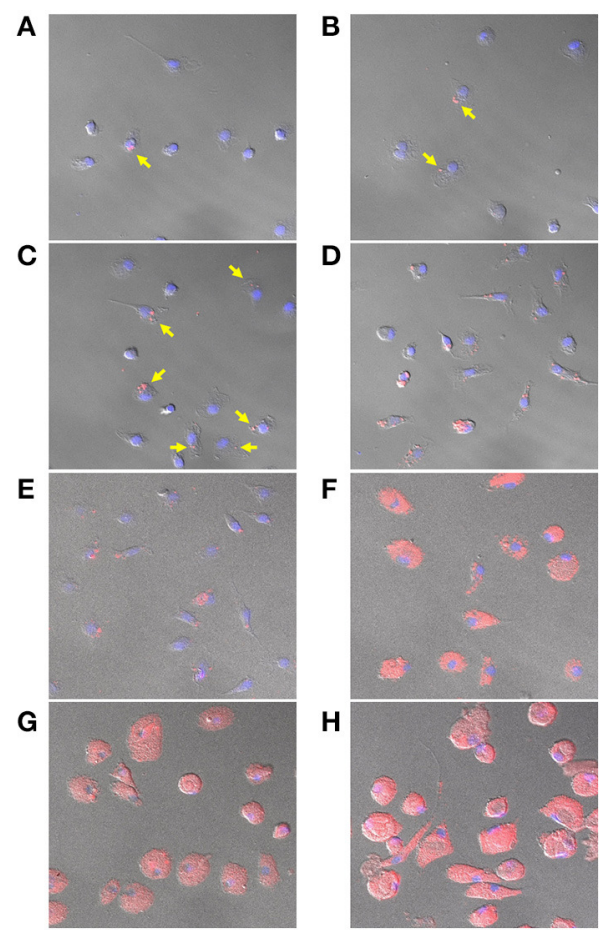

FIGURE 7 | Uptake of NP2-rhodamine by BMDM. BMDM were plated at $10^{5}$ cells per well and incubated with NP2-rhodamine for $30 \mathrm{~min}$ (A), $1 \mathrm{~h}$ (B), $2 \mathrm{~h}$ (C), 4 h (D), 6 h (E), 24 h (F), 48 h (G) or 72 h (H). Following incubation, cells were fixed with $4 \%$ PFA and slides were mounted with DAPI. Cells were analyzed by confocal fluorescence microscopy (Leica SP8). Blue = DAPI; Red $=$ rhodamine. Arrows indicate internalization of NP2-rhodamine by macrophages at early timepoints.

the morphological characteristics of NPs can predict possible interactions with living cells and uptake, intracellular localization and toxicity (Shang et al., 2014). Our results agree with previous studies (Cohen-Sela et al., 2009; McCall and Sirianni, 2013; Garbuzenko et al., 2014), demonstrating that PLGA particles produced by double emulsion presented spherical and regular shapes with smooth surfaces, regardless of modifications performed in the protocol. Following DLS, TEM and SEM characterization, P2 was selected as the better protocol for NP17-DMAG production.

We then evaluated the effects of modifications to P2: NP217-DMAG produced with $100 \mathrm{mg}$ of PLGA presented smaller sizes than $200 \mathrm{mg}$. This result stands in accordance with other authors who observed that increasing concentrations of PLGA generated larger particles (Astete and Sabliov, 2006; Rizkalla et al., 2006; Hernández-Giottonini et al., 2020). This has been observed to occur due to increased viscosity in the primary emulsion (w/o), which results in a less-efficient particle size reduction during the double emulsification process $(\mathrm{w} / \mathrm{o} / \mathrm{w})$ (Iqbal et al., 2015). Similarly, using 5\% PEG compared to $2.5 \%$ resulted in a smaller NP2-17-DMAG size. The decreased size was also observed at higher PEG concentrations in other studies (Zambaux et al., 1998; Rizkalla et al., 2006; Iqbal et al., 2015; Urbaniak and Musiał, 2019; Hernández-Giottonini et al., 2020).
Higher surfactant concentrations reduce surface tension and promote particle division during the homogenization process, thus decreasing the size of the particles formed (Keum et al., 2011; Fonte et al., 2012). This phenomenon is maintained until a saturation point is reached (Keum et al., 2011; Fonte et al., 2012; Iqbal et al., 2015; Urbaniak and Musiał, 2019).

Encapsulation efficiency (\%EE) was measured indirectly in this study. Two methods, filter/column or supernatant, were employed to separate loaded NPs and the non-encapsulated drug. We identified higher \%EE values using filter/column than the supernatant, which may be attributable to superior free drug separation by this first method. This finding is consistent with studies on nanoparticle purification efficiency (Akbulut et al., 2012; Robertson et al., 2016), demonstrating that nanoparticle separation by supernatant depends on nanoparticle size and shape, the molecular weight of the polymer, dispersion medium density, speed, and centrifugation time. The filter/column method has commonly been considered a more robust, more straightforward and efficient purification process (Robertson et al., 2016; Shah et al., 2020).

After characterizing NP2-17-DMAG, we evaluated the kinetics of drug release. We found that NP2-17-DMAG exhibited two release phases: a preliminary rapid release of $17-\mathrm{DMAG}$ lasting up to $24 \mathrm{~h}$, followed by a slow and sustained release from 24 to $72 \mathrm{~h}$. This data is similar to results obtained by Rietscher et al. (2016) and Rafiei and Azita (2017), who analyzed the release of different compounds in PLGA or PLGA-PEG NPs. PLGA NPs containing paromomycin also presented a similar release profile (Afzal et al., 2019), which was expected for polymeric NPs encapsulating hydrophilic molecules. At the initial timepoints evaluated, the drug is rapidly released due to adsorbed molecules on the surface of NPs (Hirenkumar and Steven, 2012; Kapoor et al., 2015; Rietscher et al., 2016; Mir et al., 2017; Rafiei and Azita, 2017). In contrast, at subsequent time points, a sustained and slower release occurs due to gradual degradation of the polymeric matrix and slowly release of the drug contained there (Hirenkumar and Steven, 2012; Kapoor et al., 2015; Rietscher et al., 2016; Mir et al., 2017; Rafiei and Azita, 2017).

Nanoparticle uptake was evaluated through the incubation of BMDM with NP2-rhodamine. Fluorescence microscopy analysis revealed the internalization of NPs by macrophages beginning at early times of contact with NP2-rhodamine. At later timepoints, these particles continued to be internalized and accumulation was observed in these cells' cytoplasm. These results are in agreement with other studies of professional phagocytes, including RAW 264 and J774 macrophage cell-lines and primary resident and inflammatory macrophages, which have been shown to internalize NPs at early timepoints, such as at $30 \mathrm{~min}$ of incubation (Cohen-Sela et al., 2009; Nicolete et al., 2011; Petersen et al., 2018; Couto et al., 2020; Van Hees et al., 2020).

The present study revealed that PLGA NPs containing 17DMAG prepared using a double emulsion protocol presents physical-chemical, morphological, and biological characteristics conducive to CL's treatment. Additional studies will be carried out to investigate biological and immunological effects of NP2-17-DMAG in L. braziliensis infection control both in vitro and in vivo in a future manuscript. 


\section{DATA AVAILABILITY STATEMENT}

The raw data supporting the conclusions of this article will be made available by the authors, without undue reservation.

\section{ETHICS STATEMENT}

The animal study was reviewed and approved by BALB/c mice, male or female, aged 6-12 weeks, were provided by the Gonçalo Moniz Institute (IGM/FIOCRUZ) Animal Care Facility. The animals were maintained under pathogen-free conditions, with food and water ad libitum. All procedures involving animals were conducted under the International Guiding Principles for Biomedical Research Involving Animals. The Institutional Review Board approved this study's experimental design (CEUA protocol no. 007/2020) of the Gonçalo Moniz Institute, Fiocruz, Salvador, Brazil.

\section{AUTHOR CONTRIBUTIONS}

KC, BP, VP, FF, HR, and PV conceptualized and designed the experiments and analyzed and validated the data analysis. $\mathrm{KC}$, $\mathrm{BP}, \mathrm{VP}, \mathrm{MA}, \mathrm{AP}, \mathrm{DD}$, and MC performed the experiments. KC, VP, MA, AP, HQ, and PV wrote the paper. KC, BP, HR, FF, and $\mathrm{PV}$ reviewed and edited this paper. All authors contributed to the article and approved the submitted version.

\section{REFERENCES}

Afzal, I., Sarwar, H. S., Sohail, M. F., Varikuti, S., Jahan, S., Akhtar, S., et al. (2019). Mannosylated thiolated paromomycin-loaded PLGA nanoparticles for the oral therapy of visceral leishmaniasis. Nanomedicine 14, 387-406. doi: 10.2217/nnm-2018-0038

Akbulut, O., Mace, C. R., Martinez, R. V., Kumar, A. A., Nie, Z., Patton, M. R., et al. (2012). Separation of nanoparticles in aqueous multiphase systems through centrifugation. Nano Lett. 12, 4060-4064. doi: 10.1021/nl301452x

Akhoundi, M., Kuhls, K., Cannet, A., Votýpka, J., Marty, P., Delaunay, P., et al. (2016). A historical overview of the classification, evolution, and dispersion of leishmania parasites and sandflies. PLoS Negl. Trop. Dis. 10:e0004349. doi: 10.1371/journal.pntd.0004349

Andrade-Neto, V. V., Cunha-Junior, E. F., Dos Santos Faioes, V., Martins, T. P., Silva, R. L., Leon, L. L., et al. (2018). Leishmaniasis treatment: update of possibilities for drug repurposing. Front. Biosci. Landmark 23, 967-996. doi: $10.2741 / 4629$

Astete, C. E., and Sabliov, C. M. (2006). Synthesis and characterization of PLGA nanoparticles. J. Biomater. Sci. Polym. Ed. 17, 247-289. doi: $10.1163 / 156856206775997322$

Brasil (2015). Manual de recomendações para diagnóstico, tratamento e acompanhamento de pacientes com a coinfecção Leishmania-HIV, 1st Edn. Brasília: Ministério da Saúde.

Brown, M. A., Zhu, L., Schmidt, C., and Tucker, P. W. (2007). Hsp90-From signal transduction to cell transformation. Biochem. Biophys. Res. Commun. 363, 241-246. doi: 10.1016/j.bbrc.2007.08.054

Burza, S., Croft, S. L., and Boelaert, M. (2018). Leishmaniasis. Lancet 392, 951-970. doi: 10.1016/S0140-6736(18)31204-2

Carvalho, E. M., Barral, A., Costa, J. M. L., Bittencourt, A., and Marsden, P. (1994). Clinical and immunopathological aspects of disseminated cutaneous leishmaniasis. Acta Trop. 56, 315-325. doi: 10.1016/0001-706X(94)90103-1

Chávez-Fumagalli, M. A., Ribeiro, T. G., Castilho, R. O., Fernandes, S. O. A., Cardoso, V. N., Coelho, C. S. P., et al. (2015). New delivery systems for

\section{FUNDING}

This work was supported by grants from the Bahia State Research Support Foundation (PV-Universal 422867/2016-0 and SUS0019/2014), FIOCRUZ (INOVA-46700287000), and Gonçalo Moniz Institute (PV-PROEP 400898/2013-6). PV holds a grant from $\mathrm{CNPq}(305235 / 2019-2)$. The fellowship received by $\mathrm{KC}$ was financed by Coordination for the Improvement of Higher Education Personnel-Brazil (CAPES)-Finance Code 001. This study was partially supported by the Coordination for the Improvement of Higher Education Personnel (Coordenação de Aperfeiçoamento de Pessoal de Nível Superior - CAPES) Finance Code 001. The funders had no role in study design, data collection or analysis, the decision to publish, or the manuscript's preparation.

\section{ACKNOWLEDGMENTS}

The authors would like to thank LAIPHE (FIOCRUZBA), LMN (Farmanguinhos-RJ), Electronic Microscopy Service (FIOCRUZ-BA), and Laboratory of Applied Chemistry Research (LIPAQ, SENAI CIMATEC-BA), for the support in the experiment's performance. The authors also would like to thank Carine S. A. Silva, Nathan A. A. A. Guiraud, and Gabriella B. Pita, for the realization of the initial experiments with polymeric nanoparticles, and Andris K. Walter, for English language revision and manuscript copyediting assistance.

amphotericin B applied to the improvement of leishmaniasis treatment. Rev. Soc. Bras. Med. Trop. 48, 235-242. doi: 10.1590/0037-8682-0138-2015

Chiosis, G., Vilenchik, M., Kim, J., and Solit, D. (2004). Hsp90: the vulnerable chaperone. Drug Discov. Today 9, 881-888. doi: 10.1016/S1359-6446(04)03245-3

Cohen-Sela, E., Chorny, M., Koroukhov, N., Danenberg, H. D., and Golomb, G. (2009). A new double emulsion solvent diffusion technique for encapsulating hydrophilic molecules in PLGA nanoparticles. J. Control. Release 133, 90-95. doi: 10.1016/j.jconrel.2008.09.073

Couto, P. V., Magalhães, C. P., Ferrante, M., Rebouças, J., de, S., Nguewa, P., et al. (2020). Solid lipid nanoparticles as a novel formulation approach for tanespimycin (17-AAG) against leishmania infections: Preparation, characterization and macrophage uptake. Acta Trop. 211:105595. doi: 10.1016/j.actatropica.2020.105595

Croft, S. L., Seifert, K., and Yardley, V. (2006). Current scenario of drug development for leishmaniasis. Indian J. Med. Res. 123, 399-410.

De Menezes, J. P. B., Guedes, C. E. S., Petersen, A. L. O. A., Fraga, D. B. M., and Veras, P. S. T. (2015). Advances in development of new treatment for leishmaniasis. Biomed. Res. Int. 2015, 15-18. doi: 10.1155/2015/815023

Egorin, M. J., Lagattuta, T. F., Hamburger, D. R., Covey, J. M., White, K. D., Musser, S. M., et al. (2002). Pharmacokinetics, tissue distribution, and metabolism of 17-(dimethylaminoethylamino)-17-demethoxygeldanamycin (NSC 707545) in CD 2 f 1 mice and fischer 344 rats. Cancer Chemother. Pharmacol. 49, 7-19. doi: 10.1007/s00280-001-0380-8

Erlejman, A. G., Lagadari, M., Toneatto, J., Piwien-Pilipuk, G., and Galigniana, M. D. (2014). Regulatory role of the $90-\mathrm{kDa}$-heat-shock protein (Hsp90) and associated factors on gene expression. Biochim. Biophys. Acta Gene Regul. Mech. 1839, 71-87. doi: 10.1016/j.bbagrm.2013. 12.006

Fonte, P., Soares, S., Costa, A., Andrade, J. C., Seabra, V., Reis, S., et al. (2012). Effect of cryoprotectants on the porosity and stability of insulin-loaded PLGA nanoparticles after freeze-drying. Biomatter 2, 329-339. doi: 10.4161/biom. 23246 
Formiga, F. R., Ansorena, E., De Mendonza, A. E., Imbuluzqueta, E., González, D., and Prieto, M. J. B. (2009). Nanosistemas a base de poliésteres. Nanotecnología Farm. Real. y posibilidades Farmacoter. Madrid: Real Academia Nacional de Farmacia, 41-101.

Frézard, F., Demicheli, C., and Ribeiro, R. R. (2009). Pentavalent antimonials: new perspectives for old drugs. Molecules 14, 2317-2336. doi: $10.3390 /$ molecules 14072317

Garbuzenko, O. B., Winkler, J., Tomassone, M. S., and Minko, T. (2014). Biodegradable Janus nanoparticles for local pulmonary delivery of hydrophilic and hydrophobic molecules to the lungs. Langmuir 30, 12941-12949. doi: $10.1021 / \mathrm{la} 502144 \mathrm{z}$

Graefe, S. E. B., Wiesgigl, M., Gaworski, I., Macdonald, A., and Clos, J. (2002). Inhibition of HSP90 in Trypanosoma cruzi induces a stress response but no stage differentiation. Eukaryot. Cell 1, 936-943. doi: 10.1128/EC.1.6.936-943.2002

Guswanto, A., Nugraha, A. B., Tuvshintulga, B., Tayebwa, D. S., Rizk, M. A., Batiha, G. E. S., et al. (2018). 17-DMAG inhibits the multiplication of several Babesia species and Theileria equi on in vitro cultures, and Babesia microti in mice. Int. J. Parasitol. Drugs Drug Resist. 8, 104-111. doi: 10.1016/j.ijpddr.2018.02.005

Hernández-Giottonini, K. Y., Rodríguez-Córdova, R. J., Gutiérrez-Valenzuela, C. A., Peñuñuri-Miranda, O., Zavala-Rivera, P., Guerrero-Germán, P., et al. (2020). PLGA nanoparticle preparations by emulsification and nanoprecipitation techniques: effects of formulation parameters. RSC Adv. 10, 4218-4231. doi: 10.1039/C9RA10857B

Hirenkumar, M., and Steven, S. (2012). Poly Lactic-co-Glycolic Acid (PLGA) as biodegradable controlled drug delivery carrier. Polymers (Basel). 3, 1377-1397. doi: 10.3390/polym 3031377

Hombach, A., Ommen, G., Chrobak, M., and Clos, J. (2013). The Hsp90-Stil interaction is critical for Leishmania donovani proliferation in both life cycle stages. Cell. Microbiol. 15, 585-600. doi: 10.1111/cmi.12057

Hombach, A., Ommen, G., MacDonald, A., and Clos, J. (2014). A small heat shock protein is essential for thermotolerance and intracellular survival of Leishmania donovani. J. Cell Sci. 127, 4762-4773. doi: 10.1242/jcs.157297

Iqbal, M., Valour, J. P., Fessi, H., and Elaissari, A. (2015). Preparation of biodegradable PCL particles via double emulsion evaporation method using ultrasound technique. Colloid Polym. Sci. 293, 861-873. doi: 10.1007/s00396-014-3464-9

Kapoor, D. N., Bhatia, A., Kaur, R., Sharma, R., Kaur, G., and Dhawan, S. (2015). PLGA: a unique polymer for drug delivery. Ther. Deliv. 6, 41-58. doi: $10.4155 /$ tde. 14.91

Kaye, P., and Scott, P. (2011). Leishmaniasis: complexity at the host-pathogen interface. Nat. Rev. Microbiol. 9, 604-615. doi: 10.1038/nrmicro2608

Keum, C. G., Noh, Y. W., Baek, J. S., Lim, J. H., Hwang, C. J., Na, Y. G., et al. (2011). Practical preparation procedures for docetaxel-loaded nanoparticles using polylactic acid-co-glycolic acid. Int. J. Nanomedicine 6, 2225-2234. doi: $10.2147 /$ ijn.s24547

Li, Q., Zhou, Y., Yao, C., Ma, X., Wang, L., Xu, W., et al. (2009). Apoptosis caused by Hsp90 inhibitor geldanamycin in Leishmania donovani during promastigote-to-amastigote transformation stage. Parasitol. Res. 105, 1539-1548. doi: 10.1007/s00436-009-1582-y

Lin, W. (2015). Introduction: nanoparticles in medicine. Chem. Rev. 115, 10407-10409. doi: 10.1021/acs.chemrev.5b00534

Mainardes, R. M., Gremião, M. P. D., Brunetti, I. L., Fonseca, L. M., and Khalil, N. M. (2009). Zidovudine-loaded PLA and PLA-PEG blend nanoparticles: influence of polymer type on phagocytic uptake by polymorphonuclear cells. Pharm. Nanotechnol. 98, 257-267. doi: 10.1002/jps.21406

Masmoudi, A., Hariz, W., Marrekchi, S., Amouri, M., and Turki, H. (2013). Old world cutaneous leishmaniasis: diagnosis and treatment. J. Dermatol. Case Rep. 7, 31-41. doi: 10.3315/jdcr.2013.1135

McCall, R. L., and Sirianni, R. W. (2013). PLGA nanoparticles formed by single- or double-emulsion with vitamin E-TPGS. J. Vis. Exp. e51015. doi: 10.3791/51015

Meira, C. S., and Gedamu, L. (2019). Protective or detrimental? understanding the role of host immunity in leishmaniasis. Microorganisms 7:695. doi: 10.3390/microorganisms7120695

Mir, M., Ahmed, N., and Rehman, A., ur (2017). Recent applications of PLGA based nanostructures in drug delivery. Colloids Surf. B Biointerfaces 159, 217-231. doi: 10.1016/j.colsurfb.2017.07.038
Nicolete, R., Santos, D. F. D., and Faccioli, L. H. (2011). The uptake of PLGA micro or nanoparticles by macrophages provokes distinct in vitro inflammatory response. Int. Immunopharmacol. 11, 1557-1563. doi: 10.1016/j.intimp.2011.05.014

Oryan, A., and Akbari, M. (2016). Worldwide risk factors in leishmaniasis. Asian Pac. J. Trop. Med. 9, 925-932. doi: 10.1016/j.apjtm.2016.06.021

Pallavi, R., Roy, N., Nageshan, R. K., Talukdar, P., Pavithra, S. R., Reddy, R., et al. (2010). Heat shock protein 90 as a drug target against protozoan infections: biochemical characterization of HSP90 from plasmodium falciparum and Trypanosoma evansi and evaluation of its inhibitor as a candidate drug. J. Biol. Chem. 285, 37964-37975. doi: 10.1074/jbc.M110.155317

Palma, L. C., Ferreira, L. F. G. R., Petersen, A. L., de, O. A., Dias, B. R. S., Menezes, J. P. B. de, Moreira, D. R., et al. (2019). A docking-based structural analysis of geldanamycin-derived inhibitor binding to human or Leishmania Hsp90. Sci. Rep. 9:14756. doi: 10.1038/s41598-019-51239-0

Petersen, A. L., de, O. A., Campos, T. A., Santos Dantas, D. A. dos, Rebouças, J., de, S., da Silva, J. C., et al. (2018). Encapsulation of the HSP-90 chaperone inhibitor 17-AAG in stable liposome allow increasing the therapeutic index as assessed, in vitro, on Leishmania (L) amazonensis amastigoteshosted in mouse CBA macrophages. Front. Cell. Infect. Microbiol. 8, 1-14. doi: 10.3389/fcimb.2018.00303

Petersen, A. L., de, O. A., Guedes, C. E. S., Versoza, C. L., Lima, J. G. B., de Freitas, L. A. R., et al. (2012). 17-AAG Kills intracellular Leishmania amazonensis while reducing inflammatory responses in infected macrophages. PLOS ONE 7:e49496. doi: 10.1371/journal.pone.0049496

Pratt, W. B., and Toft, D. O. (2003). Regulation of signaling protein function and trafficking by the hsp90/hsp70-based chaperone machinery. Exp. Biol. Med. 228, 111-133. doi: $10.1177 / 153537020322800201$

Quadros, H. C., Santos, L., de, M. F., Meira, C. S., Khouri, M. I., Mattei, B., et al. (2020). Development and in vitro characterization of polymeric nanoparticles containing recombinant adrenomedullin-2 intended for therapeutic angiogenesis. Int. J. Pharm. 576:118997. doi: 10.1016/j.ijpharm.2019.118997

Rafiei, P., and Azita, H. (2017). Docetaxel-loaded Plga and Plga-Peg nanoparticles for intravenous application: pharmacokinetics and biodistribution profile. Int. J. Nanomed. 12, 935-947. doi: 10.2147/IJN.S121881

Rath, S., Augusto Trivelin, L., Imbrunito, T. R., Tomazela, D. M., De Jesús, M. N., Calvo Marzal, P., et al. (2003). Antimoniais empregados no tratamento da leishmaniose: Estado da arte. Quim. Nova 26, 550-555. doi: 10.1590/S0100-40422003000400018

Rietscher, R., Czaplewska, J. A., Majdanski, T. C., Gottschaldt, M., Schubert, U. S., Schneider, M., et al. (2016). Impact of PEG and PEG-b-PAGE modified PLGA on nanoparticle formation, protein loading and release. Int. J. Pharm. 500, 187-195. doi: 10.1016/j.ijpharm.2016.01.021

Rizkalla, N., Range, C., Lacasse, F. X., and Hildgen, P. (2006). Effect of various formulation parameters on the properties of polymeric nanoparticles prepared by multiple emulsion method. J. Microencapsul. 23, 39-57. doi: 10.1080/02652040500286185

Robertson, J. D., Rizzello, L., Avila-Olias, M., Gaitzsch, J., Contini, C., Magoń, M. S., et al. (2016). Purification of Nanoparticles by Size and Shape. Sci. Rep. 6, 1-9. doi: $10.1038 /$ srep27494

Roy, N., Nageshan, R. K., Ranade, S., and Tatu, U. (2012). Heat shock protein 90 from neglected protozoan parasites. Biochim. Biophys. Acta Mol. Cell Res. 1823, 707-711. doi: 10.1016/j.bbamcr.2011. 12.003

Salvador, A., Sandgren, K. J., Liang, F., Thompson, E. A., Koup, R. A., Pedraz, J. L., et al. (2015). Design and evaluation of surface and adjuvant modified PLGA microspheres for uptake by dendritic cells to improve vaccine responses. Int. J. Pharm. 496, 371-381. doi: 10.1016/j.ijpharm.2015. 10.037

Santos, D. M., Petersen, A. L. O. A., Celes, F. S., Borges, V. M., Veras, P. S. T., and de Oliveira, C. I. (2014). Chemotherapeutic potential of 17-AAG against cutaneous Leishmaniasis caused by Leishmania (Viannia) braziliensis. PLoS Negl. Trop. Dis. 8:e3275. doi: 10.1371/journal.pntd.00 03275

Santos, D. O., Coutinho, C. E. R., Madeira, M. F., Bottino, C. G., Vieira, R. T., Nascimento, S. B., et al. (2008). Leishmaniasis treatment-a challenge that remains: a review. Parasitol. Res. 103, 1-10. doi: 10.1007/s00436-008-0943-2 
Sausville, E. A. (2004). Geldanamycin analogs. J. Chemother. 16, 68-69. doi: 10.1179/joc.2004.16.Supplement-1.68

Schopf, F. H., Biebl, M. M., and Buchner, J. (2017). The HSP90 chaperone machinery. Nat. Rev. Mol. Cell Biol. 18, 345-360. doi: 10.1038/nrm.2017.20

Scorza, B. M., Carvalho, E. M., and Wilson, M. E. (2017). Cutaneous manifestations of human and murine leishmaniasis. Int. J. Mol. Sci. 18:1296. doi: $10.3390 /$ ijms 18061296

Seifert, K. (2011). Structures, targets, and recent approaches in antileishmanial drug discovery and development. Open Med. Chem. J. 5, 31-39. doi: 10.2174/1874104501105010031

Shah, N. K., Ivone, R., Shen, J., and Meenach, S. A. (2020). A comparison of centrifugation and tangential flow filtration for nanoparticle purification: a case study on acetalated dextran nanoparticles. Particuology 50, 189-196. doi: 10.1016/j.partic.2019.06.004

Shang, L., Nienhaus, K., and Nienhaus, G. U. (2014). Engineered nanoparticles interacting with cells: size matters. J. Nanobiotechnol. 12, 1-11. doi: $10.1186 / 1477-3155-12-5$

Sidera, K., and Patsavoudi, E. (2013). HSP90 inhibitors: current development and potential in cancer therapy. Recent Pat. Anticancer. Drug Discov. 9, 1-20. doi: $10.2174 / 15748928113089990031$

Solit, D. B., and Chiosis, G. (2008). Development and application of Hsp90 inhibitors. Drug Discov. Today 13, 38-43. doi: 10.1016/j.drudis.2007.10.007

Srivastava, S., Shankar, P., Mishra, J., and Singh, S. (2016). Possibilities and challenges for developing a successful vaccine for leishmaniasis. Parasit. Vectors 9:277. doi: 10.1186/s13071-016-1553-y

Sundar, S., Mehta, H., Suresh, A. V., Singh, S. P., Rai, M., and Murray, H. W. (2004). Amphotericin B treatment for Indian visceral leishmaniasis: conventional versus lipid formulations. Clin. Infect. Dis. 38, 377-383. doi: 10.1086/380971

Urbaniak, T., and Musiał, W. (2019). Influence of solvent evaporation technique parameters on diameter of submicron lamivudine-poly- $\varepsilon$-caprolactone conjugate particles. Nanomaterials 9:1240. doi: 10.3390/nano9091240

Utreja, P., Verma, S., Rahman, M., and Kumar, L. (2020). Use of nanoparticles in medicine. Curr. Biochem. Eng. 6, 7-24. doi: 10.2174/2212711906666190724145101

Van Hees, S., Elbrink, K., De Schryver, M., Delputte, P. L., and Kiekens, F. (2020). Improving cellular uptake and cytotoxicity of chitosan-coated poly(lactic- co -glycolic acid) nanoparticles in macrophages. Nanomedicine 15, 2671-2688. doi: $10.2217 / \mathrm{nnm}-2020-0317$

Veras, P. S. T., and De Menezes, J. P. B. (2016). Using proteomics to understand how Leishmania parasites survive inside the host and establish infection. Int. J. Mol. Sci. 17:1270. doi: 10.3390/ijms17081270

Whitesell, L., and Lin, N. U. (2012). HSP90 as a platform for the assembly of more effective cancer chemotherapy. Biochim. Biophys. Acta Mol. Cell Res. 1823, 756-766. doi: 10.1016/j.bbamcr.2011.12.006
WHO (2020a). Leishmaniasis. Available online at: https://www.who.int/healthtopics/leishmaniasis\#tab=tab_1 (accessed October 14, 2020).

WHO (2020b). Weekly Epidemiological Record. Geneva: WHO, 265-280.

Wiesgigl, M., Clos, J., and Schekman, R. W. (2001). Heat shock protein 90 homeostasis controls stage differentiation in Leishmania donovani. Mol. Biol. Cell 12, 3307-3316. doi: 10.1091/mbc.12.11.3307

Wolfram, J., Zhu, M., Yang, Y., Shen, J., Gentile, E., Paolino, D., et al. (2015). Safety of nanoparticles in medicine. Curr. Drug Targets 16, 1671-1681. doi: $10.2174 / 1389450115666140804124808$

Xiao, L., Lu, X., and, M., Ruden, D. (2006). Effectiveness of Hsp90 inhibitors as anti-cancer drugs. Mini Rev. Med. Chem. 6, 1137-1143. doi: $10.2174 / 138955706778560166$

Yildirimer, L., Thanh, N. T. K., Loizidou, M., and Seifalian, A. M. (2011). Toxicological considerations of clinically applicable nanoparticles. Nano Today 6, 585-607. doi: 10.1016/j.nantod.2011.10.001

Zambaux, M. F., Bonneaux, F., Gref, R., Maincent, P., Dellacherie, E., Alonso, M. J., et al. (1998). Influence of experimental parameters on the characteristics of poly(lactic acid) nanoparticles prepared by a double emulsion method. $J$. Control. Release 50, 31-40. doi: 10.1016/S0168-3659(97)00106-5

Zhang, L., Gu, F. X., Chan, J. M., Wang, A. Z., Langer, R. S., and Farokhzad, O. C. (2008). Nanoparticles in medicine: therapeutic applications and developments. Clin. Pharmacol. Ther. 83, 761-769. doi: 10.1038/sj.clpt. 6100400

Zhang, X., and Zhang, P. (2017). Polymersomes in nanomedicine-a review. Curr. Nanosci. 13, 124-129. doi: 10.2174/15734137126661610181 44519

Zhao, R., and Houry, W. A. (2005). Hsp90: a chaperone for protein folding and gene regulation. Biochem. Cell Biol. 83, 703-710. doi: 10.1139/o 05-158

Zilberstein, D., and Shapira, M. (1994). The role of $\mathrm{pH}$ and temperature in the development of leishmania parasites. Annu. Rev. Microbiol. 48, 449-470. doi: 10.1146/annurev.mi.48.100194.002313

Conflict of Interest: The authors declare that the research was conducted in the absence of any commercial or financial relationships that could be construed as a potential conflict of interest.

Copyright $\odot 2021$ Cruz, Patricio, Pires, Amorim, Pinho, Quadros, Dantas, Chaves, Formiga, Rocha and Veras. This is an open-access article distributed under the terms of the Creative Commons Attribution License (CC BY). The use, distribution or reproduction in other forums is permitted, provided the original author(s) and the copyright owner(s) are credited and that the original publication in this journal is cited, in accordance with accepted academic practice. No use, distribution or reproduction is permitted which does not comply with these terms. 